\title{
Sequences of Prime Reciprocals. Preliminaries
}

\author{
Adam Grabowski \\ Institute of Informatics \\ University of Białystok \\ Poland
}

\begin{abstract}
Summary. In the article we formalize some properties needed to prove that sequences of prime reciprocals are divergent. The aim is to show that the series exhibits log-log growth. We introduce some auxiliary notions as harmonic numbers, telescoping series, and prove some standard properties of logarithms and exponents absent in the Mizar Mathematical Library. At the end we proceed with square-free and square-containing parts of a natural number and reciprocals of corresponding products.
\end{abstract}

MSC: 11A51 03B35

Keywords: prime factorization; sequence of prime reciprocals; harmonic number MML identifier: MOEBIUS3, version: 8.1.07 5.47.1318

\section{INTRODUCTION}

The aim of this article is to provide preliminaries needed to prove that sequences of reciprocals of prime numbers are divergent. One of the proofs (which we follow) relies on the unique decomposition of natural numbers into the square and its square-free part (similarly to Euler's 1737 original proof [4]). Essentially, it is the proof that the series exhibits log-log growth.

We start with preliminary lemmas, mainly on integrals. Section 2 introduces the notion of $n$-th harmonic number,

$$
H_{n}=\sum_{i=0}^{n} \frac{1}{i}
$$


as well as its basic properties.

We proved main steps of the proof that prime harmonic series diverges:

- the lower estimate $1+x<\exp (x)$ for the exponential function $(13)$, which holds for all $x>0$,

- $\int_{n}^{n+1} \frac{1}{x} d x<\frac{1}{n} 21$,

- $\ln (n+1)<H_{n}(22)$,

- the formula for telescoping sum (24).

Although the solution of the Basel problem states [11] that

$$
\sum_{k=1}^{n} \frac{1}{k^{2}}=\frac{\pi^{2}}{6}
$$

we proved rough upper bound, using a telescoping sum, namely, for $n \geqslant 2$,

$$
\sum_{k=1}^{n} \frac{1}{k^{2}}<\frac{5}{3}
$$

Evidently however, as in the Mizar Mathematical Library there are at least four strategies for counting the (finite) sum (and the product, similarly): to treat counted objects as

- elements of certain finite subsets;

- values of finite sequences (which is probably most frequent in MML [1], but needs recounting after concantenation or deletion of elements);

- values of the type Real_Sequence which are functions from $\mathbb{N}$ into $\mathbb{R}$ (this could be generalized into partial functions and is obviously more general than the previous one);

- bags - objects quite well developed during formalization of polynomials, in terms of bags the fundamental theorem of arithmetic is expressed in Mizar;

there is a need to propose unified approach which will be suggested to use, even if the differences between all of them are purely technical. This probably needs some revisions [6] resulting in removing existing duplications [5] of the Mizar repository. Section 6 discusses some of the details, and also introduces a Mizar functor generating bag from a given finite subset of primes. Additionally, we define two sequences of reciprocals needed for proper summing (and multiplying) later on.

The formula we still definitely need to show is

$$
\sum_{i=1}^{n} \frac{1}{i} \leqslant \prod_{p \leqslant n}\left(1+\frac{1}{p}\right) \sum_{k=1}^{n} \frac{1}{k^{2}} .
$$


The product in the formula above corresponds to the square-free part of $i$ and the sum corresponds to the square part of $i$.

In order to simplify the operations on $\mathbb{P}_{n}$ (introduced in Section 4 as the set of all primes less or equal $n$ for arbitrary $n \in \mathbb{N}$ ), we conclude our article with selected properties of reciprocals of products of sets of prime numbers.

\section{Preliminaries}

From now on $n, i, k, m$ denote natural numbers and $p$ denotes a prime number.

One can check that there exists a natural number which is non zero, square, and non trivial.

Let $Z$ be a subset of $\mathbb{R}, f$ be a partial function from $Z$ to $\mathbb{R}$, and $A$ be a subset of $\mathbb{R}$. Let us note that $f\lceil A$ is $A$-defined as a partial function from $Z$ to $\mathbb{R}$.

Let us consider a subset $Z$ of $\mathbb{R}$. Now we state the propositions:

(1) If $0 \in Z$, then $\left(\operatorname{id}_{Z}\right)^{-1}(\{0\})=\{0\}$.

(2) If $0 \notin Z$, then $\left(\operatorname{id}_{Z}\right)^{-1}(\{0\})=\emptyset$.

ProOF: $\left(\operatorname{id}_{Z}\right)^{-1}(\{0\}) \subseteq \emptyset$.

Let us consider an open subset $Z$ of $\mathbb{R}$ and a non empty, closed interval subset $A$ of $\mathbb{R}$. Now we state the propositions:

(3) If $0 \notin Z$ and $A \subseteq Z$, then $\frac{1}{\operatorname{id}_{Z}}\lceil A$ is continuous.

(4) Suppose $Z=] 0,+\infty\left[\right.$ and $A=[1, n+1]$. Then $\int_{A} \frac{1}{\operatorname{id}_{Z}}(x) d x=$ (the function $\ln )(n+1)$. The theorem is a consequence of $(2)$ and $(3)$.

(5) Suppose $Z=] 0,+\infty\left[\right.$ and $0<n$ and $A=[n, n+1]$. Then $\int_{A} \frac{1}{\operatorname{id}_{Z}}(x) d x=$ (the function $\ln )\left(\frac{n+1}{n}\right)$. The theorem is a consequence of (2) and (3).

(6) Let us consider real numbers $x, r$. Suppose $x>0$ and $r>0$. Then Maclaurin(the function exp, $]-r, r[, x)$ is positive yielding.

Proof: Set $f=$ Maclaurin(the function exp, ]-r, $r[, x)$ by [10, (8)]. For every real number $r$ such that $r \in \operatorname{rng} f$ holds $0<r$.

(7) Let us consider a summable sequence $f$ of real numbers, and a natural number $n$. If $f$ is positive yielding, then $\sum(f \uparrow(n+1))>0$.

Proof: Set $L=f \uparrow(n+1)$. For every natural number $i, 0 \leqslant L(i)$. There exists a natural number $i$ such that $i \in \operatorname{dom} L$ and $0<L(i)$. Consider $k$ being a natural number such that $k \in \operatorname{dom} L$ and $L(k)>0$. 


\section{HARMONIC Numbers}

Let $n$ be a natural number. The functor $H_{n}$ yielding a real number is defined by the term

(Def. 1) $\left(\sum_{\alpha=0}^{\kappa}\left(\operatorname{inv}_{\mathbb{N}}\right)(\alpha)\right)_{\kappa \in \mathbb{N}}(n)$.

Now we state the propositions:

(8) $H_{0}=0$.

(9) $H_{n+1}=H_{n}+\frac{1}{n+1}$.

(10) $H_{1}=1$. The theorem is a consequence of (9) and (8).

(11) $H_{2}=\frac{3}{2}$. The theorem is a consequence of (9) and (10).

\section{On Exponents And Logarithms}

Now we state the proposition:

(12) (The function $\ln )(1)=0$.

Let us consider a real number $x$. Now we state the propositions:

(13) If $x>0$, then (the function $\exp )(x)>x+1$. The theorem is a consequence of (6) and (7).

(14) If $x>0$, then (the function $\ln )(x+1)<x$.

(15) Let us consider a natural number $n$. If $n>0$, then (the function $\ln )\left(\frac{n+1}{n}\right)<\frac{1}{n}$. The theorem is a consequence of (14).

(16) Let us consider a real number $x$. Then (the function $\ln )$ ((the function $\exp (x))=x$.

(17) Let us consider real numbers $x, y$. Suppose $0<x<y$. Then (the function $\ln )(x)<($ the function $\ln )(y)$.

(18) Let us consider a non zero natural number $n$. Then (the function $\ln )(n+$ 1) $>0$. The theorem is a consequence of (12) and (17).

(19) Let us consider real numbers $x, y$. Suppose $0<x$ and $0<y$. Then (the function $\ln )(x \cdot y)=($ the function $\ln )(x)+($ the function $\ln )(y)$.

(20) Let us consider a real number $x$. Then there exists a non zero natural number $y$ such that $x<$ (the function $\ln )(($ the function $\ln )(y+1))$. The theorem is a consequence of (17) and (16).

(21) Let us consider a non empty, closed interval subset $A$ of $\mathbb{R}$, an open subset $Z$ of $\mathbb{R}$, and a non zero natural number $n$. Suppose $Z=] 0,+\infty[$ and $A=[n, n+1]$. Then $\int_{A} \frac{1}{\operatorname{id}_{Z}}(x) d x<\frac{1}{n}$. The theorem is a consequence of (2), (3), and (15). 
(22) Let us consider a non zero natural number $n$. Then (the function $\ln )(n+$ 1) $<H_{n}$.

Proof: Set $A=[1, n+1]$. Reconsider $Z=] 0,+\infty[$ as an open subset of $\mathbb{R} . A \subseteq Z \cdot \frac{1}{\operatorname{id}_{Z}}\left\lceil A\right.$ is continuous. Set $g=\frac{1}{\operatorname{id}_{Z}}$. Define $\mathcal{P}$ [natural number $] \equiv$ $\$ 1+1$

$\int_{1}^{\$_{1}+1} g(x) d x<H_{\$_{1}} \cdot \int_{A_{1}} g(x) d x<\frac{1}{1} . \mathcal{P}[1]$. For every non zero natural number $k$ such that $\mathcal{P}[k]$ holds $\mathcal{P}[k+1]$ by [2, (11), (10)], [3, (17)]. For every non zero natural number $n, \mathcal{P}[n]$.

(23) Let us consider natural numbers $n_{1}, n_{2}$. If $n_{1}{ }^{2}=n_{2}{ }^{2}$, then $n_{1}=n_{2}$.

Let $n$ be a non trivial natural number. Let us note that $n^{2}$ is non trivial.

(24) TELESCOPING SERIES:

Let us consider sequences $a, b, s$ of real numbers. Suppose for every natural number $n, s(n)=a(n)+b(n)$ and for every natural number $k, b(k)=$ $-a(k+1)$. Let us consider a natural number $n$. Then $\left(\sum_{\alpha=0}^{\kappa} s(\alpha)\right)_{\kappa \in \mathbb{N}}(n)=$ $a(0)+b(n)$.

Proof: Define $\mathcal{P}$ [natural number] $\equiv\left(\sum_{\alpha=0}^{\kappa} s(\alpha)\right)_{\kappa \in \mathbb{N}}\left(\$_{1}\right)=a(0)+b\left(\$_{1}\right)$. For every natural number $k$ such that $\mathcal{P}[k]$ holds $\mathcal{P}[k+1]$. For every natural number $n, \mathcal{P}[n]$.

(25) Let us consider sequences $f_{1}, f_{2}$ of real numbers, and a non trivial natural number $n$. Suppose for every non trivial natural number $k$ such that $k \leqslant n$ holds $f_{1}(k)<f_{2}(k)$. Then $\sum_{\kappa=1+1}^{n} f_{1}(\kappa)<\sum_{\kappa=1+1}^{n} f_{2}(\kappa)$.

Proof: Define $\mathcal{X}$ [natural number] $\equiv$ if for every non trivial natural number $k$ such that $k \leqslant \$_{1}$ holds $f_{1}(k)<f_{2}(k)$, then $\left(\sum_{\alpha=0}^{\kappa} f_{1}(\alpha)\right)_{\kappa \in \mathbb{N}}\left(\$_{1}\right)-$ $\left(\sum_{\alpha=0}^{\kappa} f_{1}(\alpha)\right)_{\kappa \in \mathbb{N}}(1)<\left(\sum_{\alpha=0}^{\kappa} f_{2}(\alpha)\right)_{\kappa \in \mathbb{N}}\left(\$_{1}\right)-\left(\sum_{\alpha=0}^{\kappa} f_{2}(\alpha)\right)_{\kappa \in \mathbb{N}}(1)$. For every non trivial natural number $n$ such that $\mathcal{X}[n]$ holds $\mathcal{X}[n+1]$. For every non trivial natural number $n, \mathcal{X}[n]$.

\section{Some Special Sequences}

The functor Reci-seq1 yielding a sequence of real numbers is defined by

(Def. 2) for every natural number $n$, it $(n)=\frac{1}{n^{2}-\frac{1}{4}}$.

Now we state the propositions:

(26) Let us consider a natural number $n$. Then $(\operatorname{Reci-seq} 1)(n)=\frac{1}{n-\frac{1}{2}}-\frac{1}{n+\frac{1}{2}}$.

(27) $\operatorname{Reci-seq1}=\operatorname{rseq}\left(0,1,1,-\frac{1}{2}\right)+-\operatorname{rseq}\left(0,1,1, \frac{1}{2}\right)$.

Let us consider a natural number $n$.

(28) $\left(\sum_{\alpha=0}^{\kappa}(\operatorname{Reci-seq} 1)(\alpha)\right)_{\kappa \in \mathbb{N}}(n)<-2$. The theorem is a consequence of (24). 
(29) $\sum_{\kappa=1+1}^{n} \operatorname{Reci-seq1}(\kappa)<\frac{2}{3}$. The theorem is a consequence of $(24)$.

Note that Basel-seq is summable.

(30) Let us consider a natural number $n$. Then $\left(\sum_{\alpha=0}^{\kappa}(\operatorname{Reci-seq1})(\alpha)\right)_{\kappa \in \mathbb{N}}(n)=$ $-2+-\frac{1}{n+\frac{1}{2}}$. The theorem is a consequence of $(24)$.

Let us consider a non trivial natural number $n$.

(31) $\sum_{\kappa=1+1}^{n} \operatorname{Basel-seq}(\kappa)<\sum_{\kappa=1+1}^{n} \operatorname{Reci-seq1}(\kappa)$.

Proof: For every non trivial natural number $k$ such that $k \leqslant n$ holds $($ Basel-seq $)(k)<($ Reci-seq1) $(k)$ by [9, (29)].

(32) $\sum_{\kappa=0}^{n} \operatorname{Basel-seq}(\kappa)<\frac{5}{3}$. The theorem is a consequence of (31) and (29).

(33) $\left(\sum_{\alpha=0}^{\kappa}(\text { Basel-seq })(\alpha)\right)_{\kappa \in \mathbb{N}}(n)<\frac{5}{3}$. The theorem is a consequence of (32).

The functor Reci-seq2 yielding a sequence of real numbers is defined by

(Def. 3) for every natural number $n$, it $(n)=1+\frac{1}{\operatorname{pr}(n)}$.

Now we state the proposition:

(34) $\sum \operatorname{Sgm}\{1\}=1$.

Let $n$ be a natural number. The functor $\mathbb{P}_{n}$ yielding a subset of $\mathbb{N}$ is defined by the term

(Def. 4) $\mathbb{P} \cap \operatorname{Seg} n$.

One can verify that $\mathbb{P}_{n}$ is finite.

Now we state the propositions:

(35) Let us consider natural numbers $m, n$. If $m \leqslant n$, then $\mathbb{P}_{m} \subseteq \mathbb{P}_{n}$.

(36) If $n+1$ is not a prime number, then $\mathbb{P}_{n+1}=\mathbb{P}_{n}$.

(37) (i) $\mathbb{P}_{0}=\emptyset$, and

(ii) $\mathbb{P}_{1}=\emptyset$.

The theorem is a consequence of (36).

(38) If $n+1$ is a prime number, then $\mathbb{P}_{n+1}=\mathbb{P}_{n} \cup\{n+1\}$.

(39) Let us consider a prime number $p$. If $p>2$, then $p+1$ is not a prime number.

(40) $\mathbb{P}_{2}=\{2\}$

(41) $n+1 \notin \mathbb{P}_{n}$.

Let $n$ be a natural number. The functor indexp $(n)$ yielding a natural number is defined by the term

(Def. 5) $\overline{\overline{\mathbb{P}_{n}}}$.

Now we state the proposition:

(42) Let us consider a natural number $n$. Then indexp $(n) \leqslant n$. 
5. Square-free and Square-containing Parts of a Natural Number

Let us consider a non zero natural number $n$. Now we state the propositions:

(43) $n=(\operatorname{TSqF} n) \cdot(n \operatorname{div} \operatorname{TSqF} n)$.

(44) $(\mathrm{SqF} n)^{2} \mid n$.

Proof: Define $\mathcal{F}$ (non zero natural number) $=\left(\prod \text { SqFactors } \$_{1}\right)^{2}$. Define $\mathcal{G}$ (non zero natural number $)=$ SqFactors $\$_{1}$. Define $\mathcal{P}$ [natural number $] \equiv$ for every non zero natural number $n$ such that support $\mathcal{G}(n) \subseteq \operatorname{Seg} \$_{1}$ holds $\mathcal{F}(n) \mid n$. For every natural number $k$ such that $\mathcal{P}[k]$ holds $\mathcal{P}[k+1] . \mathcal{P}[0]$ by [8, (20)]. For every natural number $k, \mathcal{P}[k]$.

(45) Let us consider a finite-support, natural-valued many sorted set $m$ indexed by $\mathbb{P}$, and a prime number $p$. If support $m=\{p\}$, then $\prod m=m(p)$. Proof: Consider $f$ being a finite sequence of elements of $\mathbb{C}$ such that $\prod m=\prod f$ and $f=m \cdot \operatorname{CFS}($ support $m) \cdot m \cdot\langle p\rangle=\langle m(p)\rangle$.

(46) Let us consider a non zero natural number $n$. Then $(\operatorname{SqF} n)^{2}=\operatorname{TSqF} n$. Proof: Define $\mathcal{F}$ (non zero natural number $)=\left(\prod \text { SqFactors } \$_{1}\right)^{2}$. Define $\mathcal{G}$ (non zero natural number $)=$ SqFactors $\$_{1}$. Define $\mathcal{H}$ (non zero natural number $)=\prod$ TSqFactors $\$_{1}$. Define $\mathcal{P}$ [natural number] $\equiv$ for every non zero natural number $n$ such that support $\mathcal{G}(n) \subseteq \operatorname{Seg} \$_{1}$ holds $\mathcal{F}(n)=$ $\mathcal{H}(n)$. For every natural number $k$ such that $\mathcal{P}[k]$ holds $\mathcal{P}[k+1]$. $\mathcal{P}[0]$. For every natural number $k, \mathcal{P}[k]$.

Let $n$ be a non zero natural number. Note that $n \operatorname{div}(\operatorname{SqF} n)^{2}$ is square-free as a natural number.

The functor SquarefreePart $(n)$ yielding a non zero natural number is defined by the term

(Def. 6) $n \operatorname{div} \mathrm{TSqF} n$.

Let us observe that SquarefreePart $(n)$ is square-free.

Let us consider a non zero natural number $n$. Now we state the propositions:

(47) $n=\operatorname{SquarefreePart}(n) \cdot(\operatorname{SqF} n)^{2}$. The theorem is a consequence of $(44)$ and (46).

(48) $\operatorname{support} \operatorname{PFExp}(n) \subseteq \operatorname{Seg} n$.

(49) $\operatorname{support} \operatorname{PPF}(n) \subseteq \operatorname{Seg} n$.

(50) Seg SquarefreePart $(n) \subseteq \operatorname{Seg} n$. The theorem is a consequence of (47).

(51) Let us consider non zero natural numbers $k, n$. Then $k^{2} \mid$ SquarefreePart $(n)$ if and only if $k=1$.

(52) Let us consider non zero natural numbers $m, n$. Suppose SquarefreePart $(n)=\operatorname{SquarefreePart}(m)$ and $\operatorname{TSqF} m=\operatorname{TSqF} n$. Then $m=n$. The theorem is a consequence of (47) and (46). 


\section{Generating Bags from Subsets of Prime Numbers}

Let $A$ be a finite subset of $\mathbb{P}$. The functor $A$-bag yielding a bag of $\mathbb{P}$ is defined by the term

(Def. 7) EmptyBag $\mathbb{P}+\cdot$ id $_{A}$.

Let us consider a finite subset $A$ of $\mathbb{P}$. Now we state the propositions:

(53) $\quad$ support $A$-bag $=A$.

Proof: Set $f=A$-bag. support $f \subseteq A . A \subseteq$ support $f$.

(54) If $A=\emptyset$, then $A$-bag $=$ EmptyBag $\mathbb{P}$. The theorem is a consequence of (53).

(55) Let us consider a finite subset $A$ of $\mathbb{P}$, and an object $i$. If $i \in \operatorname{support} A$-bag, then $(A-\mathrm{bag})(i)=i$. The theorem is a consequence of (53).

(56) Let us consider finite subsets $A, B$ of $\mathbb{P}$. If $A$-bag $=B$-bag, then $A=B$. The theorem is a consequence of (53).

Let $A$ be a finite subset of $\mathbb{P}$. Let us observe that $A$-bag is prime-factorizationlike and $\prod A$-bag is square-free as a natural number.

Let us consider a non zero natural number $n$ and an object $x$. Now we state the propositions:

(57) If $x \in 2^{\mathbb{P}_{n}}$, then $x$ is a finite subset of $\mathbb{P}$.

(58) If $x \in 2^{\mathbb{P}_{n}} \times \operatorname{Seg} n$, then $(x)_{1}$ is a finite subset of $\mathbb{P}$.

(59) $\operatorname{rseq}(0,1,1,0)=\operatorname{inv}_{\mathbb{N}}$.

(60) $\operatorname{indexp}(0)=0$.

Let us consider a natural number $n$.

(61) (The partial product of Reci-seq2) $(n)>0$.

(62) (The function ln) $(($ the partial product of Reci-seq2 $)(n)) \leqslant$ $\left(\sum_{\alpha=0}^{\kappa}\left(\operatorname{inv}_{\mathbb{P}}\right)(\alpha)\right)_{\kappa \in \mathbb{N}}(n)$.

Proof: Define $\mathcal{P}$ [natural number] $\equiv$ (the function $\ln )$ ((the partial product of Reci-seq 2$\left.)\left(\$_{1}\right)\right) \leqslant\left(\sum_{\alpha=0}^{\kappa}\left(\operatorname{inv}_{\mathbb{P}}\right)(\alpha)\right)_{\kappa \in \mathbb{N}}\left(\$_{1}\right)$. $\mathcal{P}[0]$. For every natural number $k$ such that $\mathcal{P}[k]$ holds $\mathcal{P}[k+1]$. For every natural number $n$, $\mathcal{P}[n]$.

(63) (The function $\ln )(($ the partial product of Reci-seq2 $)(\operatorname{indexp}(n))) \leqslant$ $\left(\sum_{\alpha=0}^{\kappa}\left(\operatorname{inv}_{\mathbb{P}}\right)(\alpha)\right)_{\kappa \in \mathbb{N}}(n)$. The theorem is a consequence of $(62)$ and (42).

The functors: Reci-Sqf and Reci-TSq yielding sequences of real numbers are defined by conditions

(Def. 8) $\operatorname{Reci-Sqf}(0)=0$ and for every non zero natural number $i, \operatorname{Reci-Sqf}(i)=$ $\frac{1}{\text { SquarefreePart }(i)}$, 
(Def. 9) $\operatorname{Reci-TSq}(0)=0$ and for every non zero natural number $i, \operatorname{Reci-TSq}(i)=$ $\frac{1}{\mathrm{TSqF} i}$,

respectively. Now we state the proposition:

(64) $\operatorname{rseq}(0,1,1,0)=$ Reci-Sqf $\cdot$ Reci-TSq.

From now on $s, s_{1}, s_{2}$ denote sequences of real numbers.

Let us consider a natural number $n$.

(65) (Reci-Sqf) $(n) \geqslant 0$.

(66) $($ Reci-TSq) $(n) \geqslant 0$.

(67) $\quad($ Basel-seq $)(n) \geqslant 0$.

(68) $\quad\left(\sum_{\alpha=0}^{\kappa}(\operatorname{rseq}(0,1,1,0))(\alpha)\right)_{\kappa \in \mathbb{N}}(n) \leqslant$

$\left(\sum_{\alpha=0}^{\kappa}(\operatorname{Reci}-\mathrm{Sqf})(\alpha)\right)_{\kappa \in \mathbb{N}}(n) \cdot\left(\sum_{\alpha=0}^{\kappa}(\operatorname{Reci}-\mathrm{TSq})(\alpha)\right)_{\kappa \in \mathbb{N}}(n)$.

Let $n$ be a non zero natural number. The functor Compose $(n)$ yielding a function from $2^{\mathbb{P}_{n}} \times \operatorname{Seg} n$ into $\mathbb{N}$ is defined by

(Def. 10) for every element $x$ of $2^{\mathbb{P}_{n}} \times \operatorname{Seg} n$ and for every finite subset $A$ of $\mathbb{P}$ and for every natural number $k$ such that $x=\langle A, k\rangle$ holds it $(x)=$ $\prod(A, 1)$-bag $\cdot k^{2}$.

Now we state the proposition:

(69) $\left(\sum_{\alpha=0}^{\kappa}(\text { Basel-seq })(\alpha)\right)_{\kappa \in \mathbb{N}}(n) \geqslant 0$.

PROOF: For every natural number $n,($ Basel-seq $)(n) \geqslant 0$ by [7, (31)].

\section{On Reciprocals of Products of Prime Numbers}

Let $n$ be a natural number. The functor $\operatorname{ReciProducts}(n)$ yielding a subset of $\mathbb{R}$ is defined by the term

(Def. 11) the set of all $\frac{1}{\prod \operatorname{Sgm} X}$ where $X$ is a subset of $\mathbb{P}_{n}$.

Let us note that ReciProducts $(n)$ is finite.

Now we state the propositions:

(70) ReciProducts $(0)=\{1\}$.

ProOF: the set of all $\frac{1}{\prod \operatorname{Sgm} X}$ where $X$ is a subset of $\mathbb{P}_{0}=\{1\}$.

(71) Let us consider a prime number $p$. If $p>2$, then $\operatorname{ReciProducts}(p+1)=$ $\operatorname{ReciProducts}(p)$. The theorem is a consequence of (39) and (36).

(72) Let us consider a natural number $p$. Suppose $p+1$ is not a prime number. Then $\operatorname{ReciProducts}(p+1)=\operatorname{ReciProducts}(p)$. The theorem is a consequence of (36).

(73) $\operatorname{ReciProducts}(1)=\{1\}$.

Proof: the set of all $\frac{1}{\prod \operatorname{Sgm} X}$ where $X$ is a subset of $\mathbb{P}_{1}=\{1\}$. 
(74) $\operatorname{ReciProducts}(2)=\left\{\frac{1}{2}, 1\right\}$.

Proof: $\{2\} \subseteq \mathbb{P}_{2}$. ReciProducts $(2) \subseteq\left\{\frac{1}{2}, 1\right\}$.

Let us consider a natural number $n$.

(75) $\operatorname{ReciProducts}(n) \subseteq \operatorname{ReciProducts}(n+1)$.

(76) Suppose $n+1$ is a prime number. Then $\operatorname{ReciProducts}(n+1)=\operatorname{ReciProdu}-$ $\operatorname{cts}(n) \cup\left\{\frac{1}{\prod \operatorname{Sgm} X}\right.$, where $X$ is a subset of $\left.\mathbb{P}_{n+1}: n+1 \in X\right\}$.

Proof: ReciProducts $(n+1) \subseteq \operatorname{ReciProducts}(n) \cup\left\{\frac{1}{\prod \operatorname{Sgm} X}\right.$, where $X$ is a subset of $\left.\mathbb{P}_{n+1}: n+1 \in X\right\}$. ReciProducts $(n) \cup\left\{\frac{1}{\prod \operatorname{Sgm} X}\right.$, where $X$ is a subset of $\left.\mathbb{P}_{n+1}: n+1 \in X\right\} \subseteq \operatorname{ReciProducts}(n+1)$.

(77) Suppose $n+1$ is a prime number. Then ReciProducts $(n+1)=\left\{\frac{1}{\prod \operatorname{Sgm} X}\right.$, where $X$ is a subset of $\left.\mathbb{P}_{n}: n+1 \notin X\right\} \cup\left\{\frac{1}{\prod \operatorname{Sgm} X}\right.$, where $X$ is a subset of $\left.\mathbb{P}_{n+1}: n+1 \in X\right\}$.

Proof: ReciProducts $(n+1) \subseteq\left\{\frac{1}{\prod \operatorname{Sgm} X}\right.$, where $X$ is a subset of $\mathbb{P}_{n}$ : $n+1 \notin X\} \cup\left\{\frac{1}{\prod \operatorname{Sgm} X}\right.$, where $X$ is a subset of $\left.\mathbb{P}_{n+1}: n+1 \in X\right\}$. $\left\{\frac{1}{\prod \operatorname{Sgm} X}\right.$, where $X$ is a subset of $\left.\mathbb{P}_{n}: n+1 \notin X\right\} \cup\left\{\frac{1}{\prod \operatorname{Sgm} X}\right.$, where $X$ is a subset of $\left.\mathbb{P}_{n+1}: n+1 \in X\right\} \subseteq \operatorname{ReciProducts}(n+1)$.

\section{REFERENCES}

[1] Grzegorz Bancerek, Czesław Byliński, Adam Grabowski, Artur Korniłowicz, Roman Matuszewski, Adam Naumowicz, and Karol Pąk. The role of the Mizar Mathematical Library for interactive proof development in Mizar. Journal of Automated Reasoning, 61(1):9-32, 2018. do1 $10.1007 /$ s10817-017-9440-6

[2] Noboru Endou, Katsumi Wasaki, and Yasunari Shidama. Definition of integrability for partial functions from $\mathbb{R}$ to $\mathbb{R}$ and integrability for continuous functions Formalızed Mathematics, 9(2):281-284, 2001.

[3] Noboru Endou, Yasunari Shidama, and Masahiko Yamazaki. Integrability and the integral of partial functions from $\mathbb{R}$ into $\mathbb{R}$. Formalized Mathematics, 14(4):207-212, 2006. doi: $10.2478 /$ v10037-006-0023-y

[4] Leonhard Euler. Variae observationes circa series infinitas. Commentarii Academiae Scientiarum Petropolitanae, 9:160-188, 1737.

[5] Adam Grabowski and Christoph Schwarzweller. On duplication in mathematical repositories. In Serge Autexier, Jacques Calmet, David Delahaye, Patrick D. F. Ion, Laurence Rideau, Renaud Rioboo, and Alan P. Sexton, editors, Intelligent Computer Mathematics, 10th International Conference, AISC 2010, 17th Symposium, Calculemus 2010, and 9th International Conference, MKM 2010, Paris, France, July 5-10, 2010. Proceedings, volume 6167 of Lecture Notes in Computer Science, pages 300-314. Springer, 2010. doi:10.1007/978-3-642-14128-7_26.

[6] Adam Grabowski and Christoph Schwarzweller. Revisions as an essential tool to maintain mathematical repositories. In M. Kauers, M. Kerber, R. Miner, and W. Windsteiger, editors, Towards Mechanized Mathematical Assistants. Lecture Notes in Computer Science, volume 4573, pages 235-249. Springer: Berlin, Heidelberg, 2007.

[7] Artur Korniłowicz and Karol Pak. Basel problem - preliminaries. Formalized Mathematics, 25(2):141-147, 2017. doi 10.1515/forma-2017-0013.

[8] Artur Korniłowicz and Piotr Rudnicki. Fundamental Theorem of Arithmetic Formalized Mathematics, 12(2):179-186, 2004. 
[9] Robert Milewski. Natural numbers. Formalized Mathematics, 7(1):19-22, 1998.

[10] Akira Nishino and Yasunari Shidama. The Maclaurin expansions, Formalized Mathematics, 13(3):421-425, 2005.

[11] Karol Pąk and Artur Korniłowicz. Basel problem. Formalized Mathematics, 25(2):149155, 2017. doi $10.1515 /$ forma-2017-0014.

Received March 27, 2018 